\title{
Beeinflusst durch eine Persönlichkeitsstörung?
}

Fragestellung: Untersucht wurden die Prävalenz von Persönlichkeitsstörungen bei Jugendlichen mit wiederholtem selbstverletzenden Verhalten sowie deren Einfluss auf selbstverletzendes Verhalten und Anpassungsfähigkeit nach einem Jahr.

Hintergrund: Bei Erwachsenen besteht eine deutliche Assoziation zwischen Persönlichkeitsstörung und selbstverletzendem Verhalten. In bisherigen Studien lag der Schwerpunkt auf der Borderline-Persönlichkeitsstörung. Ob es zulässig ist, im Jugendalter die Diagnose einer Persönlichkeitsstörung zu stellen, ist umstritten.

Patienten und Methodik: Die randomisierte kontrollierte Studie verglich die Wirksamkeit einer speziell für Jugendliche mit wiederholtem selbstverletzenden Verhalten entwickelten Gruppenpsychotherapie mit der Routineversorgung. Teilnehmer waren 366 Jugendliche (12 bis 17 Jahre) mit wiederholten Selbstverletzungen. Die Diagnose einer Persönlichkeitsstörung erfolgte mittels SKID-II mit An-
Ayodeji E, Green J, Roberts C et al. The influence of personality disorder on outcome in adolescent self-harm. Br J Psychiatry 2015; $207: 313-9$ passungen an die Altersgruppe in Bezug auf Formulierungen. Ferner wurden depressive Symptomatik, Suizidalität und das allgemeine Funktionsniveau erhoben.
Ergebnisse: $60 \%$ der durchschnittlich 14,5 Jahre alten Studienteilnehmer erfüllten die Kriterien einer Persönlichkeitsstörung. Am häufigsten waren die Borderline- und die ängstlich-vermeidende Persönlichkeitsstörung (26,6\% bzw. 25,5\%). Eine dissoziale Persönlichkeitsstörung wurde bei 18,5\% der Patienten diagnostiziert, eine depressive bei $18,2 \%$ und eine histrionische Persönlichkeitsstörung bei 12,3\%. $32 \%$ erfüllten die Kriterien von mindestens zwei Formen der Persönlichkeitsstörung. Das Risiko für selbstverletzendes Verhalten war bei den Jugendlichen mit Persönlichkeitsstörung signifikant höher als in der Gruppe ohne Persönlichkeitsstörung (60\% vs. 43\%). Die Wahrscheinlichkeit einer Verhaltensstörung war ebenfalls höher (41,9\% vs. 19\%). Hingegen war das Depressionsrisiko statistisch nicht signifikant unterschiedlich. Eine Persönlichkeitsstörung war zu allen Messzeitpunkten signifikant mit einem schlechteren Ergebnis in Bezug auf Schwere und Häufigkeit der Selbstverletzung, Anpassungsfähigkeit, Stimmung und Suizidalität assoziiert.

Schlussfolgerungen: Die Prävalenz von Persönlichkeitsstörungen bei Jugendlichen mit wiederholtem selbstverletzenden Verhalten ist hoch. Es besteht eine starke unabhängige Assoziation mit dem Ausmaß selbstverletzenden Verhaltens. Bei Jugendlichen mit selbstverletzendem Verhalten sollte daher die Möglichkeit einer Persönlichkeitsstörung in Betracht gezogen werden.

\section{- Kommentar von Jessica T. Mattivi, Mainz}

\section{Diagnosestellung mit Vorsicht}

Zum ersten Mal wurde die Prävalenz von Persönlichkeitsstörungen jedweder Art in einer Population von Jugendlichen mit wiederholtem selbstverletzenden Verhalten untersucht. $60 \%$ erfüllten die Kriterien einer Persönlichkeitsstörung, wobei diejenige vom Borderline-Typ erwartungsgemäß am häufigsten, gefolgt von ängstlich-vermeidender und dissozialer Persönlichkeitsstörung vorkam, und das Vorliegen einer solchen deutlich mit dem Ausmaß des selbstverletzenden Verhaltens zusammenhing. Die relativ unselektierte Population mit hoher Teilnehmerzahl und des geringen Loss-to-follow-up von $3 \%$ führt zu einer hohen externen Validität; somit kann von repräsentativen und generalisierbaren Ergebnissen ausgegangen werden. Zu beachten ist gleichwohl, dass die Persönlichkeitsdiagnostik lediglich zu einem einzigen Zeitpunkt erfolgte und somit keine Aussagen über die Stabilität der Diagnose über die Zeit getroffen werden kann, was jedoch gerade bei jungen Menschen mit noch nicht gefestigter Persönlichkeitsstruktur (das Durchschnittsalter der Studienteilnehmer lag bei 14,5 Jahren) erforderlich scheint. Folgerichtig weisen auch die Autoren auf die Notwendigkeit der Durchführung von Längsschnittstudien hin. Im Hinblick auf die mögliche Stigmatisierung von jungen Menschen mit einer Lebenszeitdiagnose und allen damit verbundenen Folgen sollten die Ergebnisse der vorliegenden Studie nicht dazu verleiten, bei

Jugendlichen die Diagnose einer Persönlichkeitsstörung zu stellen. Nichtsdestotrotz leistet die vorliegende Studie einen wichtigen Beitrag zur Erforschung von Persönlichkeitsstörungen in Zusammenhang mit selbstverletzendem Verhalten bei jungen Menschen. Gerade deshalb sollten sowohl Längsschnitt- als auch Therapiestudien durchgeführt werden, um die Stabilität der Diagnose und einen möglichen Nutzen von Behandlungsstrategien mit dem Schwerpunkt auf Persönlichkeitsstörungen bei Jugendlichen mit selbstverletzendem Verhalten aufzuzeigen, sodass eine grundlegende Diskussion über das Für und Wider der Diagnose einer Persönlichkeitsstörung im Jugendalter und die Einbeziehung von Persönlichkeitsaspekten in die Therapiestrategie erfolgen kann.

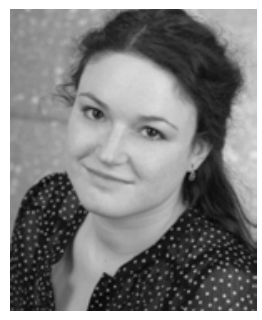

Dr. med. univ. Jessica T. Mattivi, MA, Mainz

Klinik für Psychiatrie und Psychotherapie der Universitätsmedizin Mainz

E-Mail: jessica.mattivi@unimedizin-mainz.de 